\title{
Percepción de pertinencia curricular del enfermero graduado en una universidad pública colombiana
}

\section{Perception of curricular relevance of the nurse graduated from a Colombian public university}

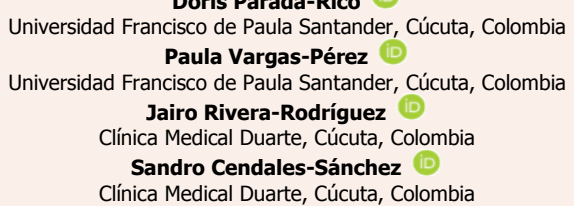

\section{Resumen}

Objetivo: Determinar en los graduados enfermeros de un programa académico de una institución universitaria pública colombiana con acreditación de alta calidad, su percepción respecto a la pertinencia de la formación académica recibida y la adquisición de competencias para su desempeño profesional. Método: Abordaje cuantitativo de tipo descriptivo transversal en el que participaron 183 graduados enfermeros de los últimos cinco años (2014-2019). Se diseñó el instrumento "evaluación del proceso formativo y su pertinencia en el desempeño profesional", con pruebas de validación facial, de contenido y confiabilidad interna, coeficiente global a de Cronbach de 0.91.Resultados:Los profesionales encuestados perciben que la pertinencia social, la integralidad y flexibilidad curricular aportan elementos relevantes para su formación, al igual que el enfoque por competencias direccionadas al desarrollo de roles enfermeros. Discusión y conclusiones: En la construcción de la malla curricular del programa, se asumen las tendencias de la profesión y disciplina como guías orientadoras para el diseño de ejes trasversales que connotan la labor profesional. Lo anterior ha configurado en el graduado una percepción de alta pertinencia de esta malla para el ejercicio laboral, aun cuando existen competencias que deben fomentarse como el análisis crítico, la socialización del conocimiento científico y la toma de decisiones autónoma disciplinar.

Palabras clave: Plan de estudios, evaluación de la educación, enfermería, acreditación, competencia.

\begin{abstract}
Objective: To determine in the nursing graduates of a Colombian public program with high quality accreditation, their perception regarding the relevance of the academic training received and the acquisition of skills for their professional performance. Method: A quantitative, descriptive, cross-sectional approach in which 183 graduate nurses from the last five years (2014-2019) participated. The instrument "evaluation of the training process and its relevance in professional performance" was designed, with tests of facial validation, content and internal reliability, Cronbach's global coefficient a of 0.91 . Results: Nursing professionals surveyed perceive that social relevance, comprehensiveness and curricular flexibility provide relevant elements for their training, as well as the focus on competencies aimed at developing nursing roles. Discussion and conclusions: In the construction of the curriculum of the program, the trends of the profession and discipline are assumed as guiding guides for the design of transversal axes that connote professional work. The above has configured in the graduate a perception of high relevance of this mesh for the work exercise, even when there are competences that should be promoted such as critical analysis, the socialization of scientific knowledge and autonomous disciplinary decision-making.
\end{abstract}

Open Access:

ISSN: $0124-2121$

E-ISSN: $2665-2420$

ARTÍCULO DE INVESTIGACIÓN CIENTÍFICA
Copyright $@$ ? By Educación y Humanismo

Editor:

Dhayana Fernández Matos Universidad Simón Bolívar

\section{Correspondencia:}

Doris Parada dorisparada@ufps.edu.co

Keywords: Curriculum, educational evaluation, nurse, accreditation, competence.

Recibido: 28-06-2020 Aceptado: $02-10-2020$ En línea desde: $16-12-2020$ 


\section{Introducción}

En el transcurso de los años, la Enfermería se ha consolidado como ciencia y disciplina, enfoca su ámbito y direccionamiento principal hacia el cuidado del individuo y colectivos durante el curso de vida. Así mismo, se inscribe en los entornos en los cuales se sitúan los sujetos con el fin de brindar atención contextualizada y acorde a los determinantes de salud implicados en ellos.

La formación de los profesionales dedicados a la Enfermería, ha sido una labor de las universidades que ofertan programas de salud; esta tarea se alinea a orientar en las dimensiones del saber, saber hacer $y$, del ser y convivir acorde a las actualizaciones de la evidencia científica, técnica, disciplinar, ética y legal, pero además fundamentada en las necesidades de un contexto situado y global (Acosta, 2012), de una sociedad cada vez más compleja y dinámica, que como plantea Mejía (2006), exige calidad.

La formación enfermera se centra en la enseñanza relacionada con el proveer cuidados desde una perspectiva científica, social, ética, política, pero esencialmente humana. Involucra a los estudiantes durante diferentes momentos de su ruta de aprendizaje en situaciones reales o simuladas, con el fin de adquirir competencias en todos los aspectos que requieren conocimientos, actitudes y habilidades para atender el proceso de salud-enfermedad y muerte en sujetos y colectivos (Casanova, 2012).

En Colombia, la Asociación Colombiana de Facultades de Enfermería, entidad que propone los lineamientos para todas las unidades académicas responsables de la formación enfermera en el país, establece que "el cuidado a la persona, la familia y las comunidades" son el eje central que dirige la formación enfermera (CTNE, 2019). Así mismo, los roles profesionales inscritos en el marco de las tendencias tanto nacionales como internacionales, deben estar direccionados al cumplimiento de funciones esenciales que incluyen la promoción de la salud, la prevención de la enfermedad y los cuidados de las personas en condición de discapacidad e incluso moribundas, y tal como lo describe el Consejo Internacional de Enfermería, estas deben fundarse en la defensa, el fomento de un entorno seguro, la investigación, la participación en la política de salud y en la gestión de los pacientes y los sistemas de salud (De-Arco \& Suarez, 2018).

El perfil del profesional en Enfermería requiere el diseño de un currículo acorde al contexto, flexible, centrado en el aprendizaje de formas y métodos que promuevan el pensamiento crítico, integral y autónomo del estudiante. Es por ello, que la actualización del currículo se propone como proceso permanente y autoevaluador que permite direccionar la formación hacia el desarrollo de competencias pertinentes para el ejercicio de la profesión.

Comprometido con esta permanente evaluación, el Programa de Enfermería de la Universidad Francisco de Paula Santander (UFPS) -institución donde se hizo el levantamiento de datos para esta investigación-, con 1702 profesionales graduados desde el año 1975, 
decidió, desde en el año 2009, inscribirse voluntariamente en el proceso de acreditación de alta calidad y seguir los lineamientos de la Política de aseguramiento de la calidad en la educación en Colombia. Es por ello por lo que esta institución educativa propone una malla curricular ajustada a las necesidades del contexto, con rutas a elegir en el Plan de Estudios, acorde a las áreas que se deseen profundizar. Además, el programa adopta tres principios orientadores: pertinencia social, flexibilidad e integralidad curricular, que intentan dar apertura a una formación trazada desde la percepción de su comunidad académica, así como de los receptores de programas y proyectos de cuidado.

En este marco de actuación, la estructura curricular del programa de Enfermería de la UFPS se definió alrededor del cuidado de la salud y la vida de los seres humanos, organizada en tres áreas de formación: ciencias básicas y clínicas, área disciplinar y área de educación complementaria. Estas áreas, a su vez se encuentran entrelazadas con ejes transversales presentes en el currículo, a saber: promoción de la salud, gestión del cuidado, ética e investigación, los cuales, según el Consejo Internacional de Enfermería (CIE), abarcan los roles principales de la profesión (De-Arco \& Suarez, 2018; Soto, Malasan, \& Barrios, 2018).

Estos ejes transversales se proponen a partir del análisis de las necesidades de formación, para desarrollar en forma efectiva conocimientos, habilidades y juicio clínico en la práctica diaria (Fuentes, Grandón, Martínez, \& Yañez, 2014). Es por ello por lo que se establece, por una parte, la gestión del cuidado como proceso heurístico para mantener y favorecer el cuidado de la persona en interacción con su contexto (Ceballos, Jara, Stiepovich, Aguilera, \& Vilchez, 2015); la promoción de la salud como un componente comprensivo y humanístico con abordajes transdisciplinares (Stein, y otros, 2009) en torno al cuidado de los sujetos y colectivos (De Albuquerque, Lumini, Ferreira, Martins, \& Ciqueto, 2016). Y por la otra parte, los ámbitos que tienen que ver con la ética y la investigación, los cuales permiten visibilizar y redimensionar el cuidado desde la perspectiva requerida por los sujetos y el contexto, a fin de responder coherentemente ante las tendencias de la disciplina y profesión, así como a las crecientes y emergentes necesidades de salud, la reorganización de los servicios y los avances científicos y tecnológicos (Mayorca, 2017).

En esta medida se espera que, al integrar los ejes transversales con las áreas principales de la malla curricular, la educación ofertada tenga las características de calidad propuesta y, asimismo, se guarde correspondencia entre las competencias previstas y desarrolladas a lo largo de la ruta de enseñanza y aprendizaje prevista en la malla curricular, el perfil del egresado y las necesidades del ámbito laboral (CTNE, 2019). Se busca entonces, generar una propuesta curricular transversal, que no se circunscriba a dimensiones únicas y en cambio favorezca la educación integral, como lo refiere Chaux (2004), citado por Cabrera-Jiménez (2020), con el fin de promover una educación que contribuya al ejercicio de la profesión con calidad, integralidad y pertinencia social. 
Lo anterior tiene tal relevancia para el diseño de los currículos que requiere una evaluación permanente, que permita la actualización de estos ejes transversales. A nivel mundial se han realizado evaluaciones que identifican de una parte, la satisfacción del estudiante con los aportes de la IE para su formación profesional (González, Lara, Pineda, \& Knofler, 2014), el logro de competencias adquiridas y su aplicación en la vida laboral (Conrado et al., 2019), pero de otra, dificultades que limitan los resultados propuestos, debido a las temporalidades cortas en las experiencias de aprendizajes, o divergencias entre lo adquirido durante la formación y los retos que enfrenta el graduado posteriormente en el ámbito de trabajo (Rojas, 2013; Barbera et al., 2015).

En Colombia han sido escasos los trabajos publicados respecto al tema, algunos de ellos resaltan la alta demanda de estudiantes y la preeminencia de programas privados para la formación enfermera (Borré, Pabón, Henao, Amaya, \& Polo, 2015), los énfasis en programas asistenciales clínicos o comunitarios, así como resultados que evidencian la necesidad de una formación integral que permita al profesional de enfermería resolver diversas situaciones presentadas en los ámbitos o roles que ocupa en su trabajo (Guerrero et al., 2010).

Así las cosas, es necesario realizar mediciones de los procesos a partir de la autoevaluación de los programas para establecer el logro de metas propuestas en la educación (Murillo, González, Urrego, 2020), especialmente en lo concerniente a la relación entre la formación del pregrado y el perfil desempeñado al ingreso laboral. De esta forma, de acuerdo a las políticas del Consejo Nacional de Acreditación (CNA), se considera que, desde la perspectiva del graduado, empleadores y usuarios de los servicios, podría identificarse el impacto de la formación y la respuesta a las necesidades del contexto a partir de la formación profesional.

Por la razón anterior, y dado que la pertinencia en el desempeño profesional, como refiere Orozco (2010), implica el aporte que cada Institución educativa (IE) hace al desarrollo de cada país, es decir, la respuesta ante las demandas sociales no sólo a nivel local, sino global como necesidad de formación (Carmona, González, \& Crespo, 2012), se hace indispensable realizar periódicamente la evaluación del programa. En este caso, el artículo presenta los resultados de un estudio diseñado con el objetivo de analizar desde la visión de los graduados, la pertinencia de la formación y competencias adquiridas frente a los roles desempeñados como enfermeros(as) en los diferentes contextos laborales tanto a nivel nacional como internacional.

Así, se develan hallazgos en el marco de la segunda renovación de acreditación de alta calidad, proceso que tiene diversas implicancias por las dinámicas que se tejen en este, pero que a su vez sirve de puente para avanzar en la identificación de fortalezas y oportunidades de mejora en el programa, para así orientar la modificación curricular y ajuste de los perfiles de egreso según los resultados. 


\section{Método}

Se realizó un estudio cuantitativo descriptivo de corte transversal, llevado a cabo dentro de las actividades establecidas en el proceso de autoevaluación del programa de enfermería de la Universidad Francisco de Paula Santander en la ciudad de Cúcuta, Colombia. Esto se propuso como una herramienta para definir aspectos de mejora en el plan de estudios relacionados con el mantenimiento de un currículo actualizado y pertinente.

La población de estudio de la investigación abarcó graduados del programa de enfermería en el periodo comprendido por los últimos cinco años (2014-2019). La muestra se estimó con un nivel de confianza de $95 \%$, error del $5 \%$ y una probabilidad de éxito de $50 \%$. Finalmente, se accedió a 183 graduados, hallados a través de redes sociales, correos electrónicos, técnica de bola de nieve, visita en su lugar de trabajo, acorde a su disponibilidad para participar en el estudio y a la autorización previa de las directivas de cada institución de trabajo. Dentro de las herramientas informáticas usadas para el diligenciamiento virtual de la encuesta, destacan Google Docs y grupos de WhatsApp.

El instrumento de recolección de información utilizado fue denominado "evaluación del proceso formativo y su pertinencia en el desempeño profesional". Este se diseñó para el estudio a partir de un proceso de validez facial y de contenido. Posteriormente, se hizo una prueba de expertos y piloto, hasta realizar finalmente la evaluación de su fiabilidad estadística como se describe en los siguientes párrafos.

El proceso de construcción del instrumento referido inició con el juicio de expertos, quienes fueron seis profesionales de enfermería, con experiencia docente, administrativa en dirección de programas, e investigativa en el ejercicio de esta disciplina, cuyos perfiles fueron Doctorado en Educación, Magíster en Enfermería, candidatas a Doctora en Ciencias Sociales, enfermeras especialistas en prácticas pedagógicas y/o currículo.

Subsiguiente al juicio de expertos, el proceso implicó ajustes de redacción y estructura de las preguntas, prueba piloto con graduados de un periodo diferente al evaluado, reajuste del contenido en una de las preguntas diseñadas, análisis de fiabilidad interna (Alfa de Cronbach) y tabulación de la información con el apoyo del paquete estadístico SPSS versión 24. Así se logró determinar un coeficiente global $a=0.91$, observándose un alto nivel de consistencia interna en el instrumento.

Se consolidó de esta forma una encuesta que consta de tres (3) grandes categorías. La primera categoría corresponde a datos de identificación que incluye doce (12) preguntas sobre datos sociodemográficos: cinco (5) preguntas abiertas y siete (7) cerradas con opción de única respuesta. La segunda categoría, denominada principios orientadores del currículo, integrada por ocho (8) preguntas, todas con única opción de respuesta en escala tipo Likert, relacionadas con la percepción del graduado respecto a la pertinencia social, integralidad y flexibilidad del currículo y el aporte que hacen en su formación como enfermero(a). 
La tercera, hace referencia a los aspectos relacionados con los ejes transversales: el eje curricular de gestión del cuidado que consta de cinco (5) preguntas; el eje curricular de promoción de la salud, con seis (6) ítems, el eje curricular de investigación, integrado por cinco (5) preguntas y el eje curricular de ética, con cinco (5) preguntas, todas con opción de única respuesta en escala de Likert. Estos ítems hacen referencia a las competencias adquiridas en los distintos ejes, y necesidades en los diferentes contextos laborales.

La escala de Likert aplicada para cada uno de los diferentes intervalos fue clasificada por los autores del instrumento de la siguiente manera: completamente de acuerdo: 5, de acuerdo:4, Ni de acuerdo ni en desacuerdo: 3, en desacuerdo: 2 y completamente en desacuerdo:1. A su vez, para la interpretación agrupada por preguntas, se clasificó de acuerdo con rangos numéricos en "alta, media y baja pertinencia", según las respuestas halladas. La información obtenida se analizó además con el software Microsoft Excel con tablas y gráficas dinámicas.

En el presente estudio se aplicaron los principios éticos expuestos por el Ministerio de Salud Pública en la Resolución 8430 de 1993, donde se establecen las normas académicas y administrativas para la investigación en seres humanos, también se fundó en la Ley 266 de 1996 la cual reglamenta los principios que rigen la profesión de enfermería y la Ley 911 de 2004, referente a la responsabilidad deontológica en el ejercicio de la enfermería en Colombia (Ministerio de Salud, 1993).

\section{Resultados}

Con respecto a la primera categoría, se pudo realizar una caracterización sociodemográfica. Se obtuvo que la edad de la mayoría de los graduados (54\%) encuestados, osciló en el rango 20 - 24 años, tal como se muestra en el gráfico $N^{\circ} 1$, probablemente por el periodo de tiempo evaluado después de su egreso institucional. Además, se identifica que en las últimas cohortes de egresados ha disminuido el tiempo en el que terminan el plan de estudio, así como disminuyen las edades de ingreso, en ese sentido, se observaron ingresos a los 16 años. 


\section{Gráfico 1.}

Distribución de graduados enfermeros según la edad.

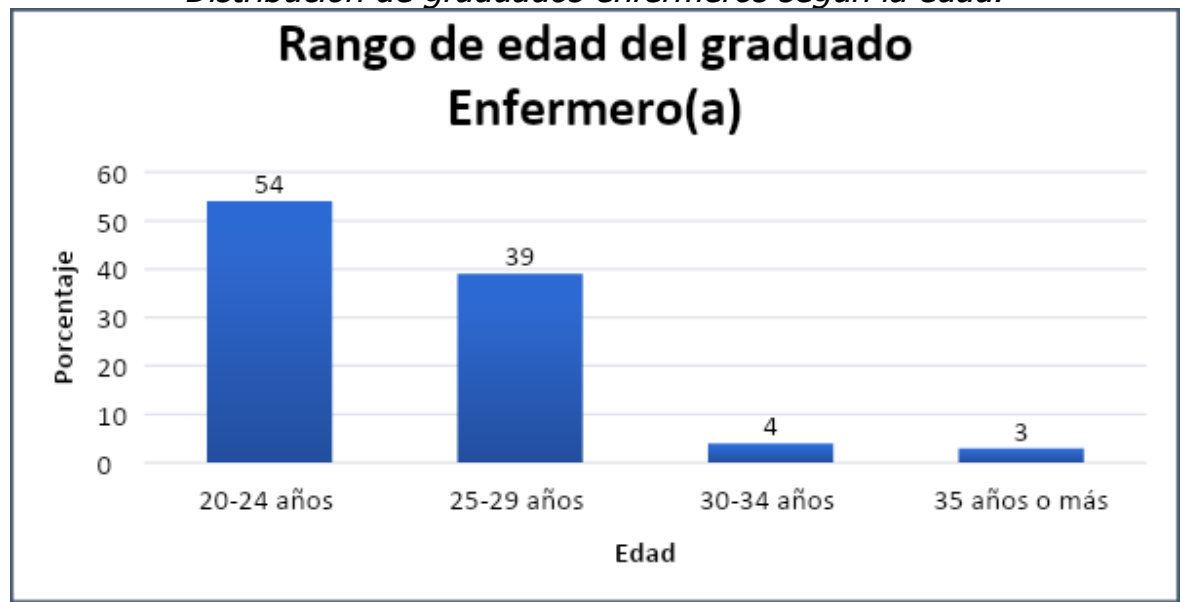

Fuente: Elaboración propia

Con respecto al género del graduado se halla que la mayoría son mujeres, muy relacionado con la división sexual de las profesiones históricamente establecida, en la que se definen las labores a realizar por mujeres y hombres, (Següel, Valenzuela, \& Sanhueza, 2015). En este caso, a pesar de la tendencia creciente en los últimos años del ingreso de hombres en esta carrera, el $77 \%$ es conformado por mujeres.

La mayoría de las personas encuestadas expresó ser soltera (74\%); el estrato socioeconómico que predomina es el 2 y 3 con $40 \%$ y $46 \%$ respectivamente, lo cual se relaciona con el tipo de entidad, pues esta es pública, de orden departamental.

Lo anterior da cuenta del mantenimiento de roles de género asignados en las profesiones, pero a su vez de las rupturas que se vislumbran en la actualidad sobre estos. Se valora la mayor proporción de jóvenes solteros, quienes privilegian continuar con ese estado civil y dar continuidad a sus trayectos académicos, relacionado probablemente con la denominada "moratoria social juvenil".

Respecto a la situación laboral, se observa que el $97 \%$ de los graduados se encuentran empleados. En relación al cargo que desempeñan, se evidencia que emerge el enfermero investigador, como se valora en el gráfico № 2 . 


\section{Gráfico 2.}

Distribución de graduados de acuerdo al cargo que desempeña.

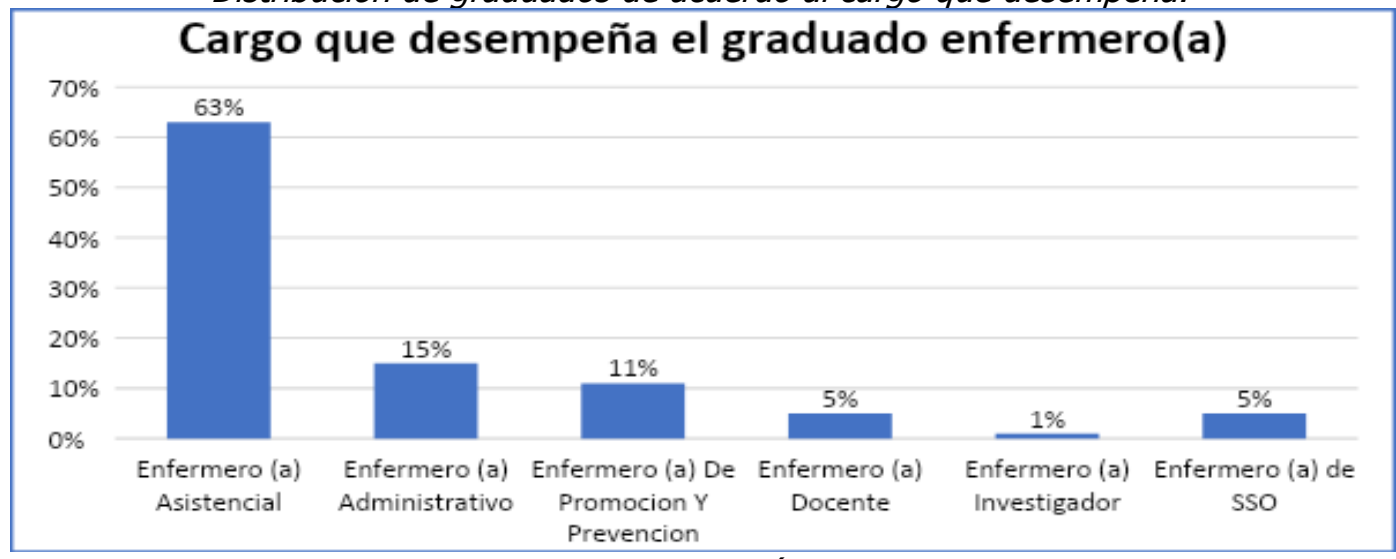

Fuente: Elaboración propia

El lugar de trabajo tanto en el ámbito nivel nacional como internacional, en instituciones públicas y privadas. Predomina la frecuencia en la ciudad de Cúcuta, como lugar de egreso educativo. En cuanto al ámbito internacional en el que se hallan las personas graduadas, se trata de Canadá, lugar donde hicieron el proceso de convalidación de estudios.

Con relación al salario actual, se encontró que la mayoría de los graduados reciben remuneración entre 2 y 3,6 salarios mínimos legales vigentes. Las clínicas privadas de Cúcuta y Medellín son las que aportan mejores salarios básicos e incrementos extras, debido a las horas adicionales de trabajo, domingos y festivos laborados

En cuanto a los reconocimientos recibidos, los más representativos son los académicos con $52 \%$ en estudios de postgrado y siguen en ese orden, los económicos, científicos, culturales y artísticos con un porcentaje de $10 \%, 4 \%, 3 \%$ y $1 \%$ respectivamente. Aunque se identifican las capacidades académicas del egresado o egresada, se evidencia un limitado abanico de posibilidades para recibir estímulos de otro tipo, especialmente culturales y/o artísticos, propuestos por el sistema de acreditación de alta calidad en Colombia como aquellos que promueven el pensamiento creativo e innovador.

\section{Principios orientadores del currículo}

Al examinar los resultados en relación a los principios orientadores del currículo y su implicancia en el desempeño laboral del graduado, se encontró que la flexibilidad, la pertinencia social e integralidad como elementos direccionadores propuestos en la malla curricular, orientan el diseño del currículo hacia la formación profesional pertinente. Estos resultados se visibilizan en las opciones de respuesta "completamente de acuerdo" y "de acuerdo".

Respecto a la pertinencia social, refieren que el currículo los preparó para desarrollar acciones de acuerdo a las necesidades del contexto a nivel nacional e internacional (91\%); la integralidad, fue valorada por el $98 \%$ de graduados, como aquel elemento que integró aspectos 
del saber disciplinar, cultural, artístico, científico y lúdico. Con respecto a la flexibilidad, el $87 \%$ respondieron que, a partir de las oportunidades como movilidad académica, la toma de electivas y líneas para profundizar, la elección de su ruta de aprendizaje a partir de cuarto semestre, o tomar un semestre de postgrado para homologar como trabajo de grado, desarrollaron capacidades para avanzar en su proyecto de formación personal y profesional. Se puede valorar los resultados por principio orientador en el gráfico $N^{\circ} 3$.

\section{Gráfico 3.}

Percepción de pertinencia según principios orientadores del currículo para la formación del enfermero.

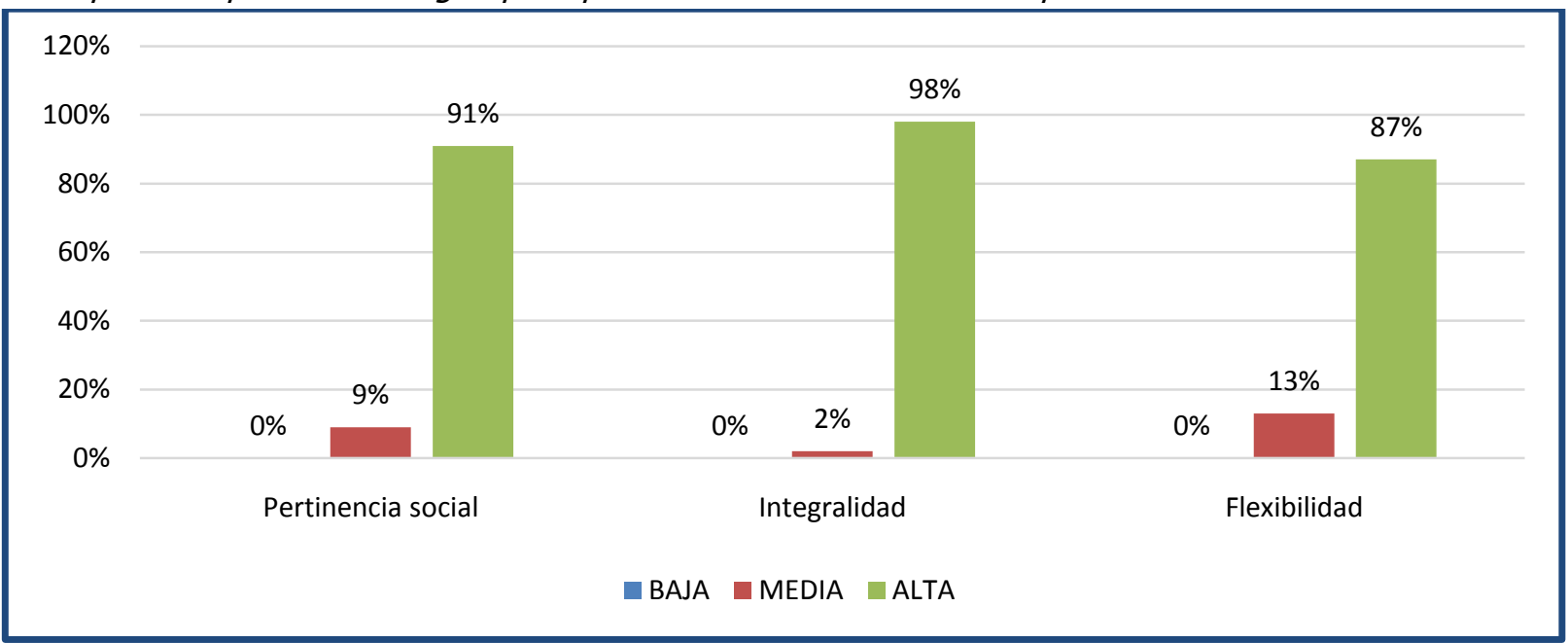

Fuente: Elaboración propia

En los resultados hallados se evidencia una tendencia creciente a percibir que el ejercicio de un rol profesional creativo, dinámico, adaptado a los diferentes contextos, que tiene en cuenta las necesidades del sujeto y colectivos de manera particular, subyace a un currículo integral, y aun cuando en orden decreciente se halla el principio de pertinencia y flexibilidad como coadyuvantes en su formación, estos se conciben por un alto porcentaje de graduados como muy relevantes en la construcción de una malla curricular enfermera.

\section{Ejes transversales del currículo}

En este aparte se indagó acerca de la percepción del graduado referente a la adquisición de competencias en los diferentes ejes que atraviesan la malla curricular, los que se relacionan con los roles del profesional de enfermería. Los hallazgos fueron los siguientes:

\section{Gestión del cuidado}

De acuerdo con la percepción que poseen los graduados sobre la adquisición de competencias en el eje curricular gestión del cuidado, se encontró el predominio de resultados que lo destacan con pertinencia elevada (94\%) para su desempeño laboral, discriminados según indicador en el gráfico $\mathrm{N}^{\circ} 4$. 
Gráfico 4. Pertinencia del eje gestión del cuidado en la formación enfermera

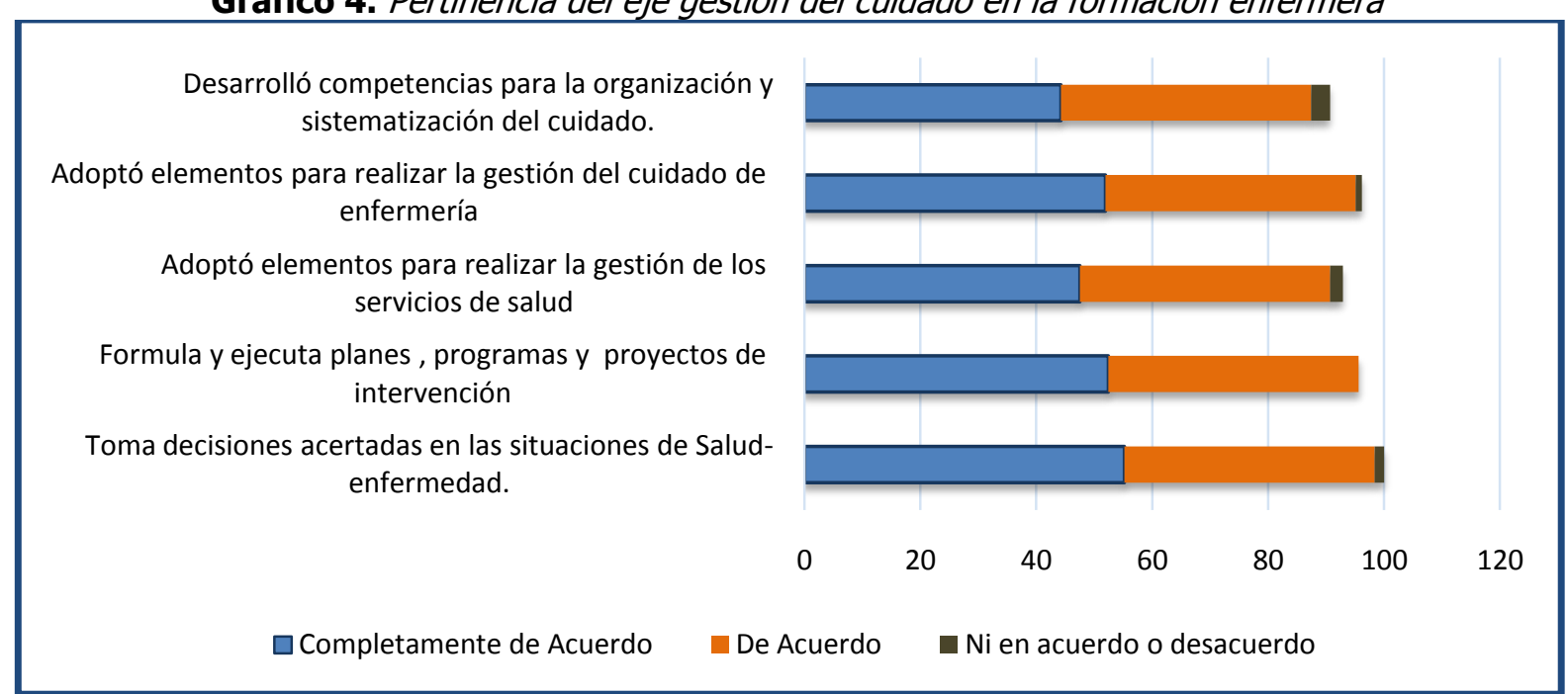

Fuente: Elaboración propia

Lo anterior refleja la percepción de pertinencia que tienen los graduados respecto del eje curricular gestión del cuidado. El 99\% percibió adquirir competencias para realizar actividades, procesos y procedimientos que implican la toma de decisiones acertadas en las situaciones de salud-enfermedad, el $97 \%$ para la formulación y desarrollo de planes, programas y proyectos de intervención en comunidades clínicas y comunitarias. El 91\% para laborar de manera efectiva en áreas que requieren la gestión administrativa de los servicios de salud.

Cabe destacar que, aunque los graduados de manera general perciben que la formación recibida les aporta elementos para desarrollar su profesión con enfoque gerencial del cuidado, llama la atención que sólo el $87 \%$ refiere la adquisición de competencias para aplicar el Proceso de Atención de Enfermería (PAE), herramienta de la disciplina eficaz para organizar y sistematizar el cuidado durante el desarrollo de las labores profesionales en el ámbito clínico y comunitario. La proporción restante en este aspecto hace referencia a que esta herramienta metodológica, tan relevante para el enfermero, no es aplicada en las instituciones de salud en las cuales laboran.

El resultado descrito en esta última parte se relaciona con una problemática dada a nivel mundial puesto que, en lo cotidiano del ejercicio profesional, en diferentes instituciones de salud, se aplica el PAE de manera incipiente o no se hace; las actividades son rutinarias y no se instaura el proceso según sus fases, sino que se prioriza el acto administrativo.

\section{Promoción de la salud}

En relación al eje de promoción de la salud y su aporte para el desempeño profesional, predominó un resultado en el que se visibiliza este eje desde la percepción del graduado como un baluarte para el desarrollo de competencias profesionales tanto a nivel clínico y comunitario. 
Se evidencia en más del $96 \%$ de los graduados la apreciación positiva de este eje, con las respuestas "completamente de acuerdo" y "de acuerdo". Refieren que este elemento curricular les permitió desarrollar conocimientos y habilidades en los diferentes ámbitos de cuidado para interactuar con la comunidad (98\%); con el equipo de salud, en el que se valora un trabajo articulado intersectorialmente (96\%). Así mismo, refieren de manera sustancial que les aporta aprendizaje en los determinantes sociales de la salud (97\%), y promueve el desarrollo de habilidades para ejecutar acciones según necesidades del contexto, como puede evidenciarse en el gráfico № 5.

Los resultados son coherentes con la tendencia a nivel nacional e internacional actual en la formación enfermera relacionada con la Atención Primaria en Salud, enfoque asumido por el programa en referencia, destacado en el desarrollo de las actividades educativas programadas.

\section{Gráfico 5.}

Pertinencia del eje transversal Promoción de la Salud, en la formación enfermera

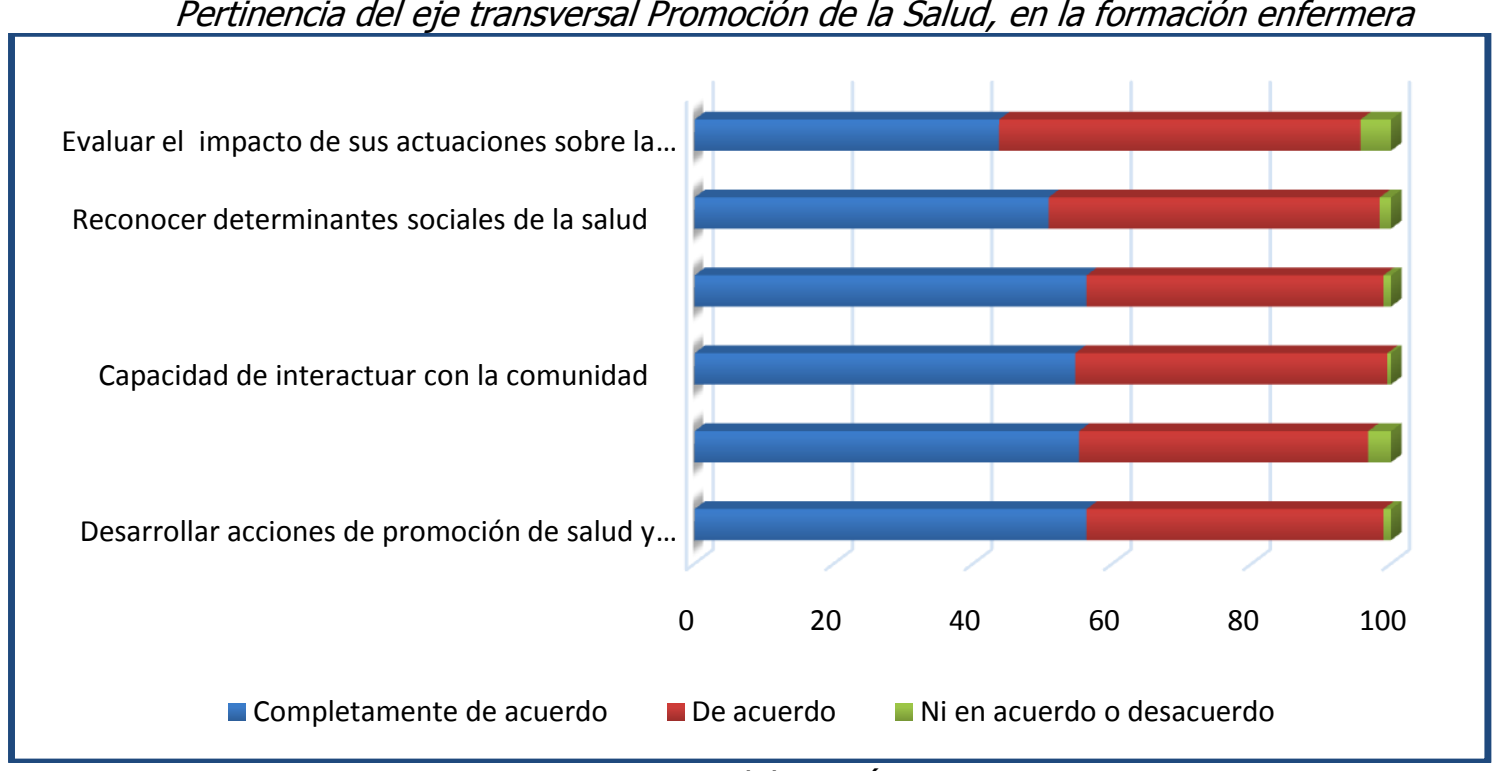

Fuente: Elaboración propia

No obstante, a pesar de las fortalezas referidas en la formación del enfermero a través de este eje, así como su pertinencia para el desempeño de la profesión, se evidencia que el aspecto relacionado con la capacidad para evaluar el impacto de los proyectos en torno a la salud de la población, sólo es referida como adquirida, "completamente de acuerdo", por el $42 \%$, relacionado a su vez con la formación recibida, pero además con los roles desempeñados por el graduado en el ejercicio de su profesión. En este caso, se debe tener en cuenta que el $63 \%$ labora en el área clínica y no llevan a cabo proyectos para evaluar el impacto de los procesos implementados. 


\section{La investigación como eje transversal en la formación}

En el análisis de este eje transversal se agruparon las respuestas "completamente de acuerdo" y "de acuerdo" para valorar los logros en estas competencias y su aplicación laboral. Se halló que el graduado considera relevante las competencias adquiridas en este ámbito, puesto que permiten ejecutar la investigación y proponer soluciones a los problemas de salud y enfermedad (99\%), realizar lectura crítica (97\%), reconocer problemas del contexto a partir del estado del arte (97\%), utilizar bases de datos científicas (96\%), como se observa en el gráfico No 6.

Se halla que para el graduado, la investigación se concibe como un valor relevante en su formación. Esta información debe ser analizada por los programas de enfermería en construcción y/o en reforma curricular, puesto que el graduado valora este eje como una herramienta primordial para su desempeño laboral.

\section{Gráfico 6.}

Pertinencia del eje transversal de investigación en la formación enfermera

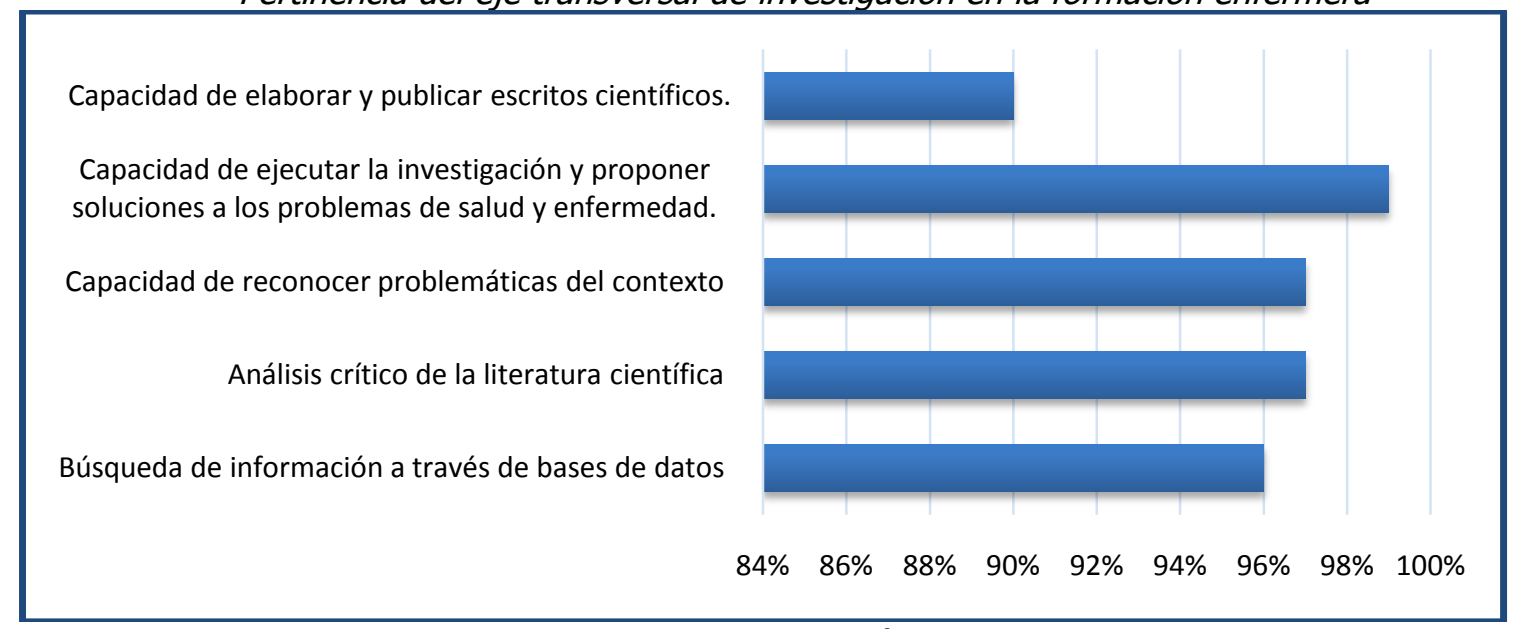

Fuente: Elaboración propia

Lo anterior refleja que los graduados perciben el eje curricular de investigación muy pertinente para su desempeño profesional, sin embargo, una de las dificultades halladas en ellos, es la escasa elaboración y publicación de artículos científicos, puesto que refieren haber alcanzado la competencia ( $90 \%)$, pero no haber realizado posteriormente esta actividad en su vida laboral, a excepción de un $4 \%$ que lo ha hecho.

\section{Eje de ética en la formación del enfermero}

Se halla que, frente a las competencias alcanzadas a través de este eje y su aplicación en el desempeño laboral, los graduados perciben, en su mayoría, haber alcanzado competencias en el fomento de los derechos humanos, el respeto por la vida, la dignidad humana, la diversidad poblacional, así como la competencia para brindar cuidado con calidad de forma humanizada en cada uno de los escenarios de cuidado, como se aprecia en el gráfico $\mathrm{N}^{\circ} 7$. 


\section{Gráfico 7.}

Pertinencia de la formación ética en el enfermero

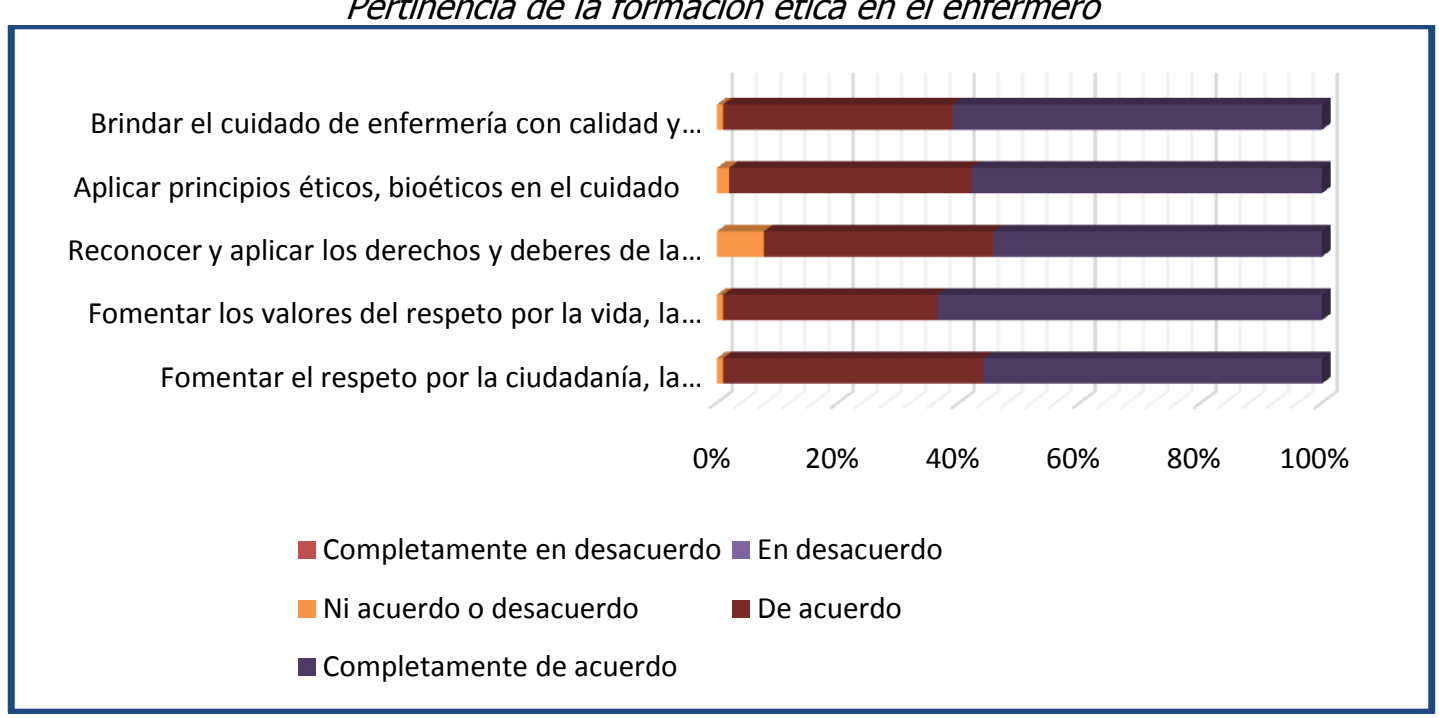

Fuente: Elaboración propia

Se puede valorar que las competencias éticas propuestas fueron alcanzadas por más del $50 \%$ de los graduados, quienes refieren estar "completamente de acuerdo" con haber logrado la adquisición de estas durante la formación y aplicarlas en su labor profesional diaria. Sin embargo, se visibiliza una disyuntiva en la competencia relacionada con la aplicación de los deberes y derechos de la profesión, puesto que, existe un porcentaje de $5 \%$ que no reconoce este logro claramente y suscribe una respuesta neutra que infiere duda ante su alcance.

En este caso puede decirse que en ocasiones ha prevalecido un halo contradictorio relacionado con el ejercicio de los deberes y derechos -no en razón a la ausencia de enseñanza de estos en la instituciones educativas formadoras u órganos colegiados o asociados- sino en el ejercicio profesional, puesto que en algunos espacios se limitan autonomías y se imponen decisiones fuera del contexto disciplinar y del Código Deontológico de la profesión.

\section{Discusión y conclusiones}

En el actual estudio, se visibiliza que desde la perspectiva del graduado emergen varios puntos que invitan a la reflexión, en este caso como enfermero de una universidad pública.

En general, el perfil del graduado del programa en estudio está acorde al del país y América Latina. Los jóvenes deciden estudiar a tempranas edades y postergar sus relaciones de pareja consensuadas o matrimoniales, por ende, el mayor número de ellos se encuentra soltero, continúan estudios de postgrado (OIT, 2009) y laboran casi inmediatamente al salir de su pregrado. Estos datos coinciden con el Observatorio Laboral para la Educación (MEN, 2020), el cual presenta que entre el $90,2 \%$ y $94,7 \%$ de graduados de programas de enfermería en el 
periodo estudiado, inician su cotización en salud a partir del primer empleo y mantienen estructuras que permiten engancharse al mercado laboral (MEN, 2019). En el programa analizado se observa un porcentaje de enganche laboral superior a esta cifra (97\%), debido al hecho de egresar de un programa de alta calidad, como lo refieren los graduados.

Referente a los salarios, se evidencia que existe una gran brecha entre los pagos realizados por entidades públicas y privadas, especialmente cuando para laborar en estas últimas deben cancelar pagos de seguridad social y afiliación a riesgos laborales de manera personal, y no gozan de periodos vacacionales remunerados. No obstante, los participantes de esta investigación se encuentran en el rango salarial que a nivel nacional reciben estos profesionales, como lo evidencia el Observatorio Laboral para la Educación (MEN, 2020). Además, estos datos coinciden con los hallados por Roque et al., (2018) en Ecuador, en el que se identifican pagos entre 2 a 4 salarios mínimos de ese país, a los graduados de enfermería.

Con respecto al objetivo orientado a identificar la pertinencia de la formación recibida en el programa y su aplicación en el ejercicio profesional, se halla en general, que la percepción de este grupo supera el valor dado por los graduados a nivel nacional en Colombia, quienes, según el informe del Observatorio Laboral para la Educación, lo consideran pertinente en el 67\%- 80\% de los casos (MEN, 2019). En este caso, los graduados definen haber alcanzado las competencias necesarias para su desempeño en los diferentes roles profesionales, y valoran con alto grado de pertinencia, la formación recibida en el pregrado. Estos resultados son concordantes con estudios a nivel nacional e internacional (Medina \& Do Prado, 2009; Latrach, Febré, Demandes, Araneda, \& González, 2011; Bautista, Jiménez, \& Meza, 2012; López, Barahona, Preciado, Favela, \& Cuen, 2015; Carrera, García, Aguilar, \& Vicente, 2017; Roque et al., 2018), en los que existe una alta congruencia de los aspectos desarrollados en su formación y el ejercicio de su rol profesional.

Cabe destacar que al plantear esta investigación la discriminación por principios orientadores, se pudo identificar elementos básicos de análisis, puesto que los aspectos relacionados con la flexibilidad, integralidad y pertinencia social del programa visibilizaron tanto indicadores positivos como oportunidades de mejora. Se valora que, a pesar de que el graduado refiere que estos elementos aportaron de manera significativa a su formación y propiciaron la adquisición de competencias para su vida personal y profesional, se requiere mejorar la difusión de las diversas opciones que tienen como estudiantes y así mismo proponer otros espacios de interacción para desarrollos culturales y artísticos.

Los hallazgos anteriores respecto a la pertinencia social del programa como principio orientador de la malla curricular y su contribución para el desempeño laboral del enfermero, valorado desde la perspectiva del graduado, coincide con los resultados dados desde la visión del empleador quien manifiesta que los graduados del Programa de Enfermería de la UFPS responden a las necesidades de la institución, de los servicios, y diseñan propuestas acordes a las características del contexto (Salazar, Rangel, \& Ramírez, 2012). 
En relación a la percepción de pertinencia que tiene el graduado respecto a los ejes transversales que permean la malla curricular y su aporte para la formación de competencias y roles profesionales, se halla que estos están alineados a los perfiles requeridos en los distintos ámbitos laborales: la gestión del cuidado, la promoción de la salud, la ética e investigación han sido claves para formar un profesional que pueda dar respuesta en las distintas instancias del cuidado, niveles de complejidad y zonas geográficas.

Es importante destacar que se requiere profundizar y apoyar más desde el eje de investigación la participación del estudiante en procesos de escritura y publicación de artículos, puesto que sólo un pequeño grupo de graduados refiere que esta labor ha sido aplicada en su ejercicio profesional, lo cual implica demás la escasa visibilidad que da el grupo enfermero en estudio a sus trabajos investigativos realizados, tal como lo refieren otros autores (Bautista, Jiménez, \& Meza, 2012; Barbera et al., 2015).

\section{Referencias}

Acosta, A. (2012). En la encrucijada de la glocalización. Revista Latinoamericana Polis, 1-17. http://journals.openedition.org/polis/7039

Barbera, M., Cecagno, D., Seva, A., Heckler, H., López, M., y Soler, L. (2015). Formación académica del profesional de enfermería y su adecuación al puesto de trabajo. Revista Latinoamericana Enfermagem, 23(3), 404-410. https:10.1590/0104-1169.0432.2569

Bautista, L., Jiménez, S., \& Meza, J. (2012). Desempeño laboral de los enfermeros (a) egresados de la Universidad Francisco de Paula Santander. Revista Ciencia Y Cuidado, 9(2), 64-70. https://doi.org/10.22463/17949831.340

Borré, Y., Pabón, Y., Henao, L., Amaya, K., y Polo, K. (2015). Caracterización de los programas de enfermería en Colombia. Investigaciones Andina, 17(31), 1418-1432. https://www.redalyc.org/articulo.oa?id=2390/239040814011

Cabrera, M. (2020). Relevancia de las competencias ciudadanas en construcción de civilidad en educación superior. Educación y Humanismo, 22(38), 1-24. https://orcid.org/0000-00031030-8626

Carmona, B., González, S., \& Crespo, S. (2012). Opinión de los egresados del plan de estudios de la licenciatura en Enfermería de la Facultad de Estudios Superiores ZaragozaUniversidad Nacional Autónoma de México. Enfermería Global, 11(28), 180-191. http://scielo.isciii.es/scielo.php?script=sci_arttext\&pid=S1695-

$61412012000400011 \& \operatorname{lng}=$ es\&nrm=iso 
Carrera, V., García, A., Aguilar, E., \& Vicente, G. (2017). Percepción de pertinencia del plan de estudios de los egresados de complementario de Licenciatura en Enfermería a distancia: UJAT. Memorias del Encuentro Internacional de Educación a Distancia, (5), 1-12. http://www.udgvirtual.udg.mx/remeied/index.php/memorias/article/view/274

Casanova, M. (2012). El diseño curricular como factor de calidad educativa. Revista Iberoamericana sobre Calidad, Eficacia y Cambio en Educación, 10(4), 6-20, http://www.rinace. net/reice/numeros/arts/vol10num4/art1.pdf

Ceballos, P., Jara, A., Stiepovich, J., Aguilera, P., y Vilchez, V. (2015). La gestión del cuidado: una función social y legal de la enfermería chilena. Revista Electrónica Enfermería Actual en Costa Rica, 29, 1-12. http://dx.doi.org/10.15517/revenf.v0i29.19733

Conrado, A., Silva, F., Barbosa, D., Alves, V., Francisco, B., y DaCosta, F. (2019). Perfil de egresados del curso de enfermería: competencias e inserción profesional. Revista Latinoamericana Enfermagen, 27e. https://doi.org/10.1590/1518-8345.3222.3205

CTNE. (2019). Plan Nacional de Enfermería 2020-2030. Propuesta preliminar. https://www.oceinfo.org.co/difusion/normatividad/send/4-normatividad/147-propuestaplan-nacional-de-enfermeria-2020-2030

De Albuquerque, R., Lumini, M., Ferreira, M., Martins, T., \& Ciqueto, H. (2016). Una mirada sobre la promoción de salud y la prevención de complicaciones: diferencias de contextos. Revista LatinoAmericana de Enfermagen, (24e), 1182-9. http://dx.doi.org/10.1590/1518-8345.0860.2749

De-Arco, O., y Suarez, Z. (2018). Rol de los profesionales de enfermería en el sistema de salud colombiano. Universidad y Salud, 20(2), 171-182. http://dx.doi.org/10.22267/rus.182002.121

Fuentes, P., Grandón, K., Martínez, D., \& Yañez, O. (2014). Competencias para la enfermera/o en el ámbito de gestión y administración: desafíos actuales de la profesión. Aquichan, 14(1), 79-99. 10.5294/aqui.2014.14.1.7

González, M., Lara, A., Pineda, S., y Knofler, S. (2014). Perfil de ingreso de los alumnos de Enfermería, Facultades de Estudios Superiores Iztacala y Zaragoza. Enfermería Universitaria, 11(1), 11-18. 10.1016/S1665-7063(14)72659-4

Guerrero, V., López, Á., Mavisoy, S., Rodríguez, K., Rosero, C., y Acosta, M. (2010). Desempeño del profesional de enfermería en el área asitencial en una institución de salud de la ciudad de Pasto-Nariño. Unimar, 28(33), 43-50. http://editorial.umariana.edu.co/revistas/index.php/unimar/article/view/191/168

Latrach, C., Febré, N., Demandes, I., Araneda, J., \& González, I. (2011). Importancia de las 
competencias en la formación de enfermería. Aquichan, 11(3). https://aquichan.unisabana.edu.co/index.php/aquichan/article/view/2020/2581

López, J., Barahona, N., Preciado, M., Favela, A., \& Cuen, F. (2015). Evaluación de competencias del profesional de enfermería que labora en hospitales públicos y privados. Revista Electrónica Enfermería actual en Costa Rica, (27), 1-15. http://dx.doi.org/10.15517/revenf.v0i27.16057

Mayorca, C. (2017). La ética en la investigación de Enfermería. Rev enferm Herediana, 10(2), 71-72. https://doi.org/10.20453/renh.v10i2.3359

Medina, J., y DoPrado, M. (2009). El curriculum de enfermería como prototipo de Tejné: racionalidad instrumental y tecnológica. Texto \& Contexto Enfermagem, 18(4), 617-626. https://doi.org/10.1590/S0104-07072009000400002

Mejía, M. (2006). Educación(es) en la(s) globalización(es). Entre el pensamiento único y la nueva crítica Tomo II. Desde Abajo. Fondo Editorial UCH.

Ministerio de Educación Nacional. (2019). Seguimiento a graduados de educación superior 2017. https://ole.mineducacion.gov.co/1769/articles-380398_recurso_1.pdf

Ministerio de Educación Nacional. (2020). Observatorio Laboral para la Educación. https://ole.mineducacion.gov.co/portal/

Ministerio de Salud. ( 4 de octubre de 1993). Resolución Nº08430 de 1993. Bogotá: MinSalud.

Murillo, G., González, C., Urrego, D. (2020). Transformación del sistema de aseguramiento de la calidad de la Educación Superior en Colombia 2019-2020. Educación y Humanismo, 23(38), 1-27. 10.17081/eduhum.22. 38.3541

Organización Internacional del Trabajo. (2009). La igualdad de género como eje del trabajo decente. Ginebra: OIT.

Orozco-Silva, L. (2010). Calidad académica y relevancia social de la educación superior en América Latina. Revista Iberoamericana de Educación Superior, 1(1), 24-36.

Rojas, L. (2013). Pertinencia del plan de estudios de la Licenciatura en Enfermería de la Universidad de Costa Rica con el contexto socio-laboral. Revista Electrónica de Enfermería Actual en Costa Rica, (24), 1-16. http://www.revenf.ucr.ac.cr/pertinencia.pdf

Roque, Y., Gafas, C., Herrera, A., Salazar, Y., Betacourt, C., y Figueredo, K. (2018). Pertinencia de la formación académica de enfermería. Educación Médica, 19(2), 73-78. https://doi.org/10.1016/j.edumed.2017.04.010

Salazar, L., Rangel, S., \& Ramírez, M. (2012). Evaluación del desempeño profesional del 
enfermero egresado durante los años del 2002 al 2011 de la Universidad Francisco de Paula Santander por parte del empleador en la ciudad San José de Cúcuta [Tesis de pregrado, Universidad Francisco de Paula Santander].

Següel, F., Valenzuela, S., \& Sanhueza, O. (2015). El trabajo del profesional de enfermería: revisión de la literatura. Ciencia y Enfermería, 21(2), 11-20. http://dx.doi.org/10.4067/S0717-95532015000200002

Soto, P., Malasan, P., \& Barrios, S. (2018). La Educación en Salud, un elemento central del cuidado de Enfermería. Revista Médica Clínica, 29(3), 288-300. 10.1016/j.rmclc.2018.05.001

Stein, M., Martins, L., Manfrini, G., Azevedo, S., Becker, S., \& Schlindwein, B. (2009). Conceitos de saúde e doença ao longo da história sob o olhar epidemiológico e antropológico. Rev. enferm. UERJ, 17(1), 111-117. 